\title{
Statistical comparison of the observed and synthetic hydrogen Lyman line profiles in solar prominences
}

\author{
S. Gunár ${ }^{1}$, P. Schwartz ${ }^{1}$, B. Schmieder ${ }^{2}$, P. Heinzel ${ }^{1}$, and U. Anzer ${ }^{3}$ \\ 1 Astronomical Institute, Academy of Sciences of the Czech Republic, 25165 Ondřejov, Czech Republic \\ e-mail: gunar@asu.cas.cz \\ 2 Observatoire de Paris, Section de Meudon, 92195 Meudon Principal Cedex, France \\ 3 Max-Planck-Institut für Astrophysik, Karl-Schwarzschild-Strasse 1, 85740 Garching, Germany
}

Received 6 October 2009 / Accepted 23 January 2010

\section{ABSTRACT}

\begin{abstract}
Aims. We analyse a unique set of prominence SOHO/SUMER Lyman spectra by comparing it with synthetic spectra obtained by $2 \mathrm{D}$ multi-thread prominence fine-structure models.

Methods. We employed a novel statistical approach to the analysis of the observed and synthetic Lyman spectra. We compared the statistical distributions of the line properties of the observed and synthetic Lyman spectra using a set of four statistical criteria. Results. We demonstrate the very good agreement between the observed Lyman spectra and synthetic spectra obtained by modelling. Conclusions. Our set of statistical criteria is well-suited to analyses of the prominence Lyman spectra because of its sensitivity to a number of different parameters governing the conditions in the prominence fine structures.
\end{abstract}

Key words. Sun: filaments, prominences - radiative transfer - line: profiles - techniques: spectroscopic

\section{Introduction}

With progress in solar observations from space, reliable UV and EUV data on prominences have become available. This offered new possibilities for spectroscopic investigation of the prominence plasma and magnetic fields. Hydrogen Lyman lines and continuum observations, in particular, represent a unique probe to the structure and physical properties of quiescent prominences. A substantial amount of prominence spectral data in Lyman lines was provided by SUMER (Solar Ultraviolet Measurements of Emitted Radiation) UVspectrograph (Wilhelm et al. 1995) onboard SOHO (Solar and Heliospheric Observatory). The SOHO prominence observations were reviewed by Patsourakos \& Vial (2002); see also a review of the prominence fine structures by Heinzel (2007).

The interpretation of the prominence observations in the Lyman lines requires sophisticated models of the prominence plasma in the magnetic field and complex non-LTE radiative transfer computations. The first non-LTE 1D model in magnetohydrostatic (MHS) equilibrium based on the KippenhahnSchlüter magnetic structure (Kippenhahn \& Schlüter 1957) with multi-level radiative transfer computations was developed by Heasley \& Mihalas (1976). Subsequent development of similar isothermal-isobaric 1D models was led by a number of authors (Gouttebroze, Fontenla, Heinzel, Vial). The substantial influence of the prominence-corona transition region (PCTR) on the Lyman-line intensities was demonstrated by Anzer \& Heinzel (1999), by showing that a departure from isothermal models was needed. Thus Heinzel \& Anzer (2001) constructed the 2D nonLTE model of an individual prominence fine-structure thread in the MHS equilibrium with the PCTR, which exhibits different shapes along and across the magnetic field. This considerably affects the resulting Lyman line profiles. Line profiles obtained across the magnetic field (narrow PCTR with a steep temperature gradient) tend to be significantly reversed, while profiles obtained along the magnetic field (wide PCTR with gradually increasing temperature) are usually purely emission. This corresponds to the assumptions of Heinzel et al. (2001) and is further corroborated by Heinzel et al. (2005) and Schmieder et al. (2007). The importance of the multi-thread prominence fine-structure models is shown by Gunár et al. (2007), who used a trial-and-error method to reproduce the observed Lyman spectrum. Furthermore, Gunár et al. (2008) added random line-of-sight (LOS) macroscopic velocities to each thread of the multi-thread model, thus showing that even relatively low LOS velocities (of the order of $10 \mathrm{~km} \mathrm{~s}^{-1}$ ) have a significant effect on the asymmetries of the Lyman line profiles.

In the present work we compare the observed Lyman spectra with the synthetic spectra resulting from multi-thread modelling of the prominence fine structures based on Gunár et al. (2008). However, instead of comparing individual Lyman line profiles we analyse statistical distributions of line properties of the observed and synthetic spectra characterized by following statistical criteria: the integrated intensities of the Lyman lines, the Lyman decrement (ratios of the integrated intensities of Lyman lines to Lyman- $\beta$ ), the red-to-blue peak intensity ratio that characterizes the asymmetries of the line profiles, and finally the centre to mean peak intensity ratio characterizing the depth of the profile reversals. For statistical purposes, we use large sets of observed and synthetic spectra.

The paper is organized as follows. Section 2 gives details regarding the observed spectra. Section 3 briefly presents the multi-thread models and provides details on the input parameters of the models used in the present study. Section 4 shows results of the comparison of the observed and synthetic spectra for four statistical criteria, and Sect. 5 contains discussion and our conclusions. 


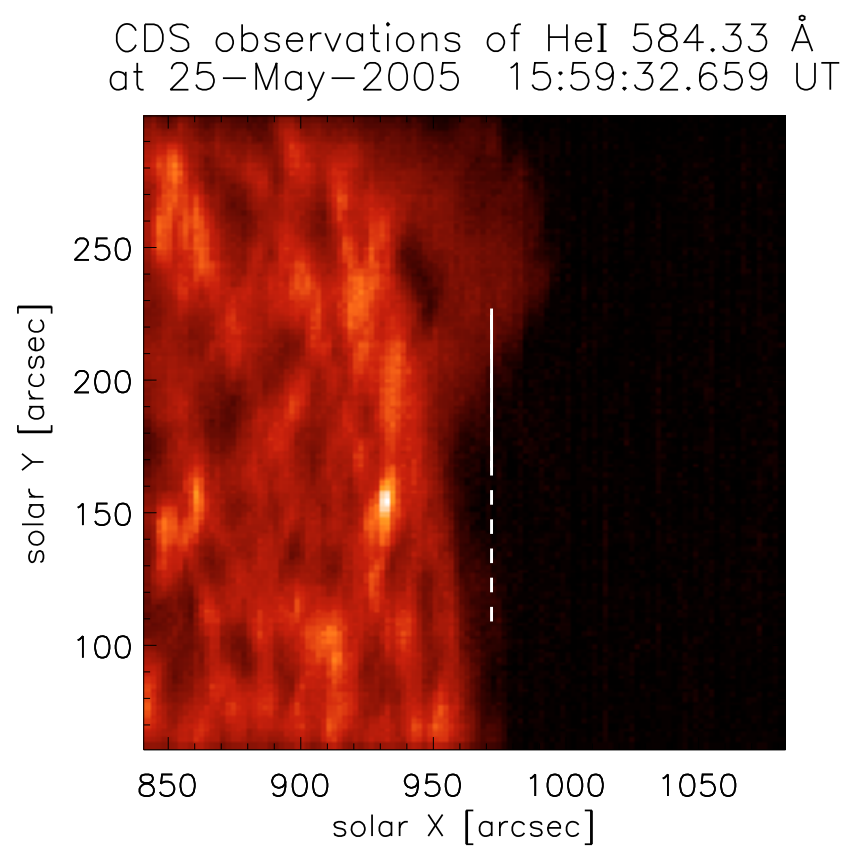

Fig. 1. Raster obtained by SOHO/CDS (Coronal Diagnostic Spectrometer) (Harrison et al. 1995) in the He I $584.33 \AA$ line on May 25, 2005 between 15:59:33 and 16:52:26 UT. The position of the SUMER slit is marked by the vertical bar crossing the southern leg of the prominence. Full-line part of the bar marks the working part of detector $\mathrm{A}$.

\section{Observed spectra}

The observed spectra was obtained by the SUMER on May 25 and 26,2005 . The observations were made in the sit-and-stare mode with the spectrograph slit crossing the southern part of the prominence, as shown in Fig. 1. The observed data set consists of the whole hydrogen Lyman line series obtained using four wavelength windows taken with the detector A. The exposure time of $115 \mathrm{~s}$ was used for each observation. The Lyman- $\alpha$ line was obtained outside the attenuator in the first spectral window. The Lyman- $\beta$ line was obtained in the second, the Lyman- $\gamma$ with the Lyman- $\delta$ in the third, and higher Lyman lines up to the Lyman continuum were obtained in the fourth spectral window.

The observations were made in three time blocks with the correction for solar rotation applied between them. The first block was made on May 25, 2005 between 16:03:15 UT and 20:40:59 UT, the second block of observations was made between 20:46:27 UT and 23:01:26 UT, and the third block was made between 23:04:54 UT on May 25, 2005 and 01:21:51 UT on May 26, 2005. The number of observations in each spectral window in a given observational block is indicated in Table 1.

Three different values of the detector voltage were applied in different times of Lyman- $\alpha$ observations. However, we did not detect any significant influence of the different voltages on our statistical analyses of the observed spectra, so we took all Lyman- $\alpha$ observations into account regardless of the different voltages. An example of the observed spectra is shown in Fig. 2.

The observed spectra were reduced and calibrated using standard SolarSoft procedures for the SUMER data. The following procedures were applied in this order: decompression of binary data saved in IDL-save files, dead-time correction, flat-fielding, local-gain correction, and correction for geometrical distortion of the detector. Finally, the data were calibrated to $\mathrm{W} / \mathrm{m}^{2} / \mathrm{sr} / \AA$ units with the radiometry procedure

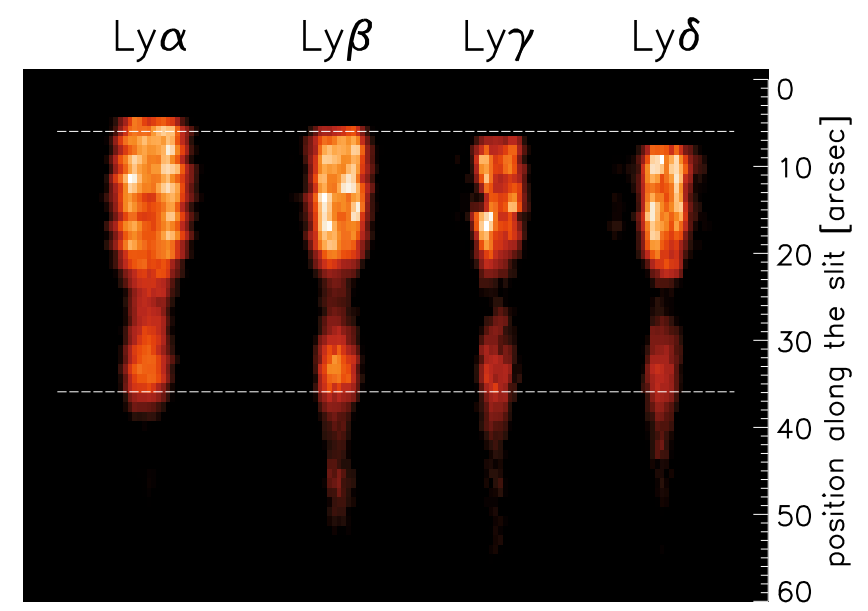

Fig. 2. Example of the observed spectra obtained on May 25, 2005 by SOHO/SUMER. Lyman- $\alpha$ line was observed at 16:22:45, Lyman- $\beta$ at 16:20:27, and Lyman- $\gamma$ and Lyman- $\delta$ at 16:18:18 UT. Dashed lines show the portion of the spectra we used in our analyses.

Table 1. Number of observations in each spectral window within each time block.

\begin{tabular}{cccc}
\hline \hline Spectral window & P1 & P2 & P3 \\
\hline Lyman- $\alpha$ & 25 & 12 & 13 \\
Lyman- $\beta$ & 40 & 20 & 20 \\
Lyman- $\gamma$ and Lyman- $\delta$ & 39 & 20 & 20 \\
Lyman-5 to continuum & 10 & 10 & 10 \\
\hline P1 - 16:03:15 UT to 20:40:59 UT \\
P2 - 20:46:27 UT to 23:01:26 UT \\
P3 - 23:04:54 UT to 01:21:51 UT \\
\hline
\end{tabular}

(Schühle 2003, private communication). For detailed technical informations about the instrument, corrections, and procedures see http://www.mps.mpg.de/projects/soho/ sumer/text/webluca/ch_iust.html and references therein. The lines were identified with the SUMER spectral catalogue (Curdt et al. 2001). The spectral image on the detector is inclined with respect to the detector's horizontal axis due to different orientations of the grating and the detector. Thus the spectra of different lines are vertically shifted with respect to each other. In addition there is a vertical shift due to the displacement of the slit image on the detector caused by the nonlinearity of the grating focus mechanism (Schühle 2003). The shifts caused by both effects were computed for all the lines using the SolarSoft procedure delta_pixel and these corrections were taken into account in our modelling.

For the purpose of this statistical analysis we used only the observed spectra between pixels 5 and 35 for Lyman- $\alpha$ to Lyman- $\delta$ (Fig. 2). In this part all the lines have high intensities, so the region is certainly part of the observed prominence. We consider only the Lyman- $\alpha$ to Lyman- $\delta$ lines because there are fewer observations of the higher members of the hydrogen Lyman line series, and they exhibit higher levels of noise.

\section{Synthetic spectra}

To obtain the synthetic Lyman lines, we employ the same multi-thread prominence fine-structure models as in Gunár et al. (2008). These consist of sets of identical 2D single-thread models (developed by Heinzel \& Anzer 2001) without any mutual radiative interaction. Single $2 \mathrm{D}$ threads are vertically infinite and 
are in MHS equilibrium. The empirical temperature structure is characterized by a steep gradient of the temperature across the magnetic field lines and by a gradual rise of the temperature along the magnetic field from the central minimum temperature to the PCTR temperatures at the boundaries. For the detailed description of these 2D thread models, we refer the reader to Heinzel \& Anzer (2001) and Heinzel et al. (2005).

The 2D fine-structure threads are arranged along individual magnetic field lines perpendicular to the LOS. Each thread is randomly shifted in its position along given magnetic field line and has randomly assigned LOS velocity $\xi^{(n)}$ (Fig. 3; for example of one particular realization see also Fig. 2 of Gunár et al. 2008). The emerging intensity from such multi-thread model at a given wavelength and position along the length of the foremost thread (thread $N$ ) in the observers frame is described by a formula

$$
\begin{aligned}
I_{\text {total }}(\lambda)= & {\left[\ldots\left[\left[I_{\lambda^{(1)}} \times \exp \left(-\tau_{\lambda^{(2)}}\right)+I_{\lambda^{(2)}}\right] \times \exp \left(-\tau_{\lambda^{(3)}}\right)+I_{\lambda^{(3)}}\right]\right.} \\
& \left.\times \ldots+I_{\lambda^{(N-1)}}\right] \times \exp \left(-\tau_{\lambda^{(N)}}\right)+I_{\lambda^{(N)}},
\end{aligned}
$$

where $I_{\lambda^{(n)}}$ and $\tau_{\lambda^{(n)}}$ are defined as

$I_{\lambda^{(n)}} \equiv I\left(\lambda-\Delta \lambda^{(n)}\right)$

$\tau_{\lambda^{(n)}} \equiv \tau\left(\lambda-\Delta \lambda^{(n)}\right)$.

Here $\Delta \lambda^{(n)}$ is the Doppler shift for the thread $n$ corresponding to its LOS velocity $\xi^{(n)}$ given by

$\Delta \lambda^{(n)}=\lambda_{0} \frac{\xi^{(n)}}{c}$,

where $\lambda_{0}$ represents the line-centre wavelength of a given spectral line, and $\xi^{(n)}$ is the LOS velocity of thread $n$. Positive values of $\xi^{(n)}$ represent velocities oriented towards the observer. The intensity $I_{\lambda^{(n)}}$ represents the intensity of radiation emerging from a single thread $n$ obtained by solving the non-LTE radiative transfer problem within a 2D fine-structure thread illuminated by the solar surface (see Heinzel \& Anzer 2001). The mutual radiative interaction of the individual threads is not taken into account.

For the purpose of this statistical study, we have also modified the formal solver to compute the emerging radiation for angles different from the directions across or along the magneticfield lines. For any given realization of the multi-thread model (similar to the one shown in Fig. 3 but with the LOS inclined to the direction perpendicular to the magnetic field), the positions of intersections of the LOS with the surface of each 2D thread were calculated. Then the distribution of the source function and the opacity along the inclined LOS for each thread was obtained using a linear interpolation from the source function and opacity distributions obtained by solving the non-LTE radiative transfer problem within a single $2 \mathrm{D}$ fine-structure thread illuminated by the solar surface. The optical thickness along the inclined LOS was computed for each thread by integrating the interpolated opacity along the geometrical intersection of the LOS and the $2 \mathrm{D}$ thread. The emerging intensity from each $2 \mathrm{D}$ thread in the direction of the inclined LOS $\left(I_{\lambda^{(n)}}\right.$ from Eq. (1)) was computed by formal solution of the radiative transfer using the quadrature formula of Mihalas et al. (1978). The total emerging intensity in the direction of the inclined LOS was then computed using Eq. (1). Again, no mutual radiative interaction of the threads is taken into account. Note that in the case of the inclined LOS, the actual values of the LOS velocities are those of the velocities with LOS perpendicular to the magnetic field reduced by appropriate inclination factor.

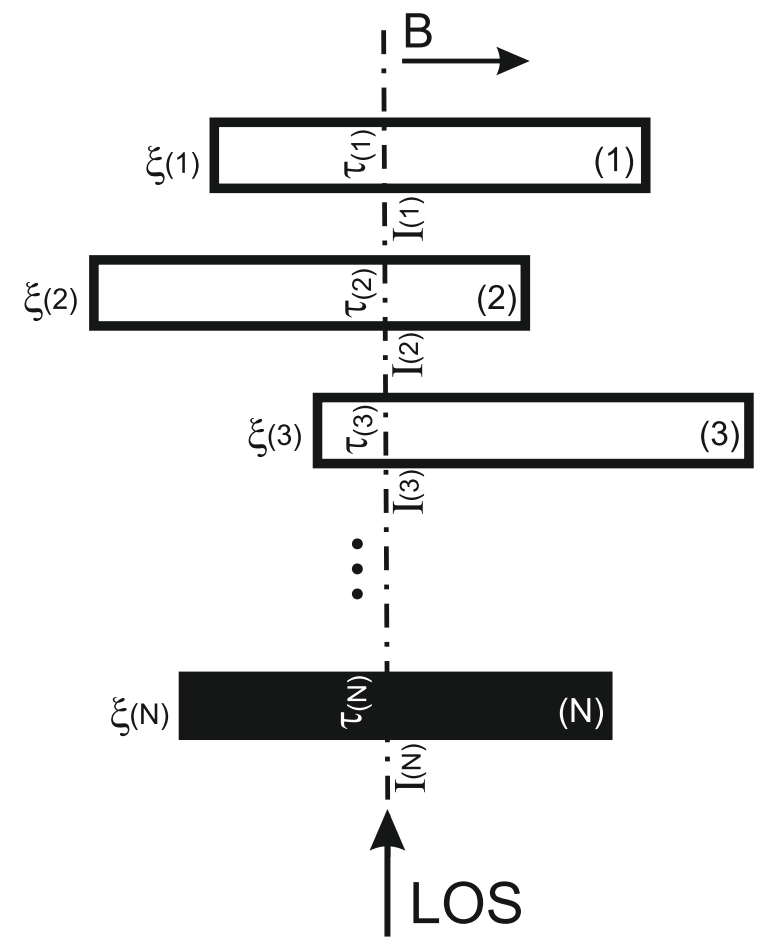

Fig. 3. Scheme of the multi-thread model with $N$ randomly shifted 2D threads and randomly assigned LOS velocities $\left(\xi^{(n)}\right) . B$ is the horizontal component of the magnetic field.

\begin{tabular}{|c|c|c|}
\hline \multirow{2}{*}{ Label } & \multicolumn{2}{|l|}{ Set of input parameters } \\
\hline & \multicolumn{2}{|l|}{ Common to all models } \\
\hline MODEL1 & \multirow{3}{*}{ 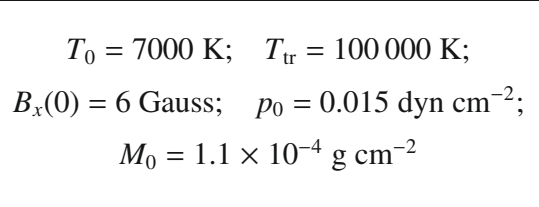 } & $\begin{array}{l}\gamma_{1}=10 \\
\gamma_{2}=60\end{array}$ \\
\hline VAR1 & & $\begin{array}{c}\gamma_{1}=5 \\
\gamma_{2}=30\end{array}$ \\
\hline VAR2 & & $\begin{array}{l}\gamma_{1}=10 \\
\gamma_{2}=30\end{array}$ \\
\hline
\end{tabular}

Table 2. List of input parameters of the 2D single-thread models.

\subsection{Input parameters}

It is not the aim of this paper to look for the prominence model with the best agreement between particular synthetic and observed Lyman line profiles. Therefore, we focus our analyses on variation of several input parameters of our multi-thread models centered on the model labeled hereafter as MoDEL1. This model was found to have the best agreement between observed and synthetic Lyman line profiles for the same prominence by Gunár et al. (2007) and it was used for the analysis of the Lyman line asymmetries by Gunár et al. (2008). The other two models presented in this study are minor variations of the reference MODEL1 and are thus named VAR1 and VAR2.

The input parameters of a given multi-thread model fall into two distinctive categories. First category involves the physical parameters of the individual 2D fine structure thread, while the second category represents the layout of the threads within the multi-thread model. The input parameters of the individual 2D threads are described in Heinzel \& Anzer (2001), so here we only give their brief outline (see Table 2). The parameter $B_{x}(0)$ represents the magnetic field strength in the middle of the thread, $M_{0}$ gives the maximum column density, and $p_{0}$ is the boundary pressure. The temperature structure of the individual $2 \mathrm{D}$ thread is characterized by minimum central temperature $T_{0}$, maximum 
boundary temperature $T_{\mathrm{tr}}$, and also by the shape of the PCTR with two different gradients of the temperature along and across the magnetic field lines. These temperature gradients can be described by exponents $\gamma_{1}$ and $\gamma_{2}$, where $\gamma_{2}$ prescribes very steep gradient across the field lines and $\gamma_{1}$ represents gradual rise of the temperature along the field lines from the centre of the thread towards its boundaries (see Heinzel \& Anzer 2001).

The layout of the threads of the multi-thread model is characterized by the total number of threads, by the distance between them, by their relative shifts in the direction along the magnetic field, and by the LOS velocities of the individual threads. In our present analysis we assume the total number of threads to be 10 and the distance between individual threads to be $1000 \mathrm{~km}$ for all models. For the maximum relative shift of individual threads with respect to the foremost thread, we assume that it is half of the length of the threads. Relative shifts of threads and their actual LOS velocities are generated randomly for each realization of given multi-thread structure. Thus the only input parameter describing the layout of the threads, which varies between individual multi-thread models, is the interval of the randomly generated LOS velocities. As was shown by Gunár et al. (2008), even relatively low LOS velocities (of the order of $10 \mathrm{~km} \mathrm{~s}^{-1}$ ) can have a considerable effect on the asymmetries of the Lyman line profiles. In the present investigation we consider three intervals of the LOS velocities, $\langle-5,5\rangle,\langle-10,10\rangle$, and $\langle-20,20\rangle \mathrm{km} \mathrm{s}^{-1}$.

\subsection{Synthetic data sets}

To obtain sufficiently extensive synthetic data sets of the Lyman line profiles, we computed one hundred realizations of each multi-thread model. Each realization has the same input parameters for individual 2D threads and the same total number of threads. We assume all threads of a given multi-thread model to be identical. The multi-thread realizations differ in the sets of the positional shifts of individual threads with respect to the foremost thread and in the actual LOS velocities of the individual threads. Then each synthetic data set consists of 83 equidistantly spaced positions along the foremost thread of the multi-thread model (one quasi slit spectrum) for each of one hundred realizations of the given multi-thread model, representing one hundred quasi observations at different times. Note that one pixel on the SUMER slit corresponds to approximately $700 \mathrm{~km}$ and the distance between two positions of our quasi slit is approximately $340 \mathrm{~km}$.

All resulting synthetic Lyman line profiles are convolved with the instrumental profile of the SUMER A detector. This instrumental profile is a Gaussian with a standard deviation $\sigma$ equivalent to one SUMER A detector spectral pixel, which corresponds to $0.043 \AA$. This agrees with the results of Chae et al. (1998), who measured the instrumental FWHM (full width at half maximum) of the SUMER A detector to be approximately 2.3 detector pixels. The FWHM for the Gaussian distribution is equal to approximately $2.4 \sigma$.

\section{Statistical criteria}

As shown by Heinzel et al. (2005) and Schmieder et al. (2007), the inclination of the LOS with respect to the orientation of the magnetic field plays an important role in the analysis of the Lyman line profiles. The line profiles obtained with the LOS perpendicular to the magnetic field exhibit significant reversals, even in the case of high members of the Lyman line series.
On the other hand, the Lyman line profiles obtained with the LOS oriented along the magnetic field tend to be unreversed, especially the higher members of the Lyman series. This effect can be explained by a difference in the shape of the PCTR when observed across and along the magnetic field lines. The LOS perpendicular to the magnetic field intersects a very narrow PCTR with a steep gradient of the temperature. The LOS oriented along the magnetic field lines intersects much wider PCTR with a shallower decrease in the temperature from the prominence fine structure boundary towards its centre. Therefore, one might be able to infer the inclination of the LOS with respect to the orientation of the magnetic field from the shape of the observed Lyman line profiles.

The observed spectra we use in the present study mostly incorporates profiles with the significant reversals, which implies that the LOS during these observations was more-or-less perpendicular to the predominant magnetic field inside the observed prominence. Therefore, we focus our statistical analyses on the multi-thread models with the LOS inclined no more than $60^{\circ}$ from that perpendicular to the magnetic field.

The multi-thread models with the LOS close to the one parallel to the magnetic field show increasing predominance of purely emission Lyman line profiles, which is in a good agreement with the previous theoretical works. In the following analyses, we assume the LOS to be perpendicular to the magnetic field if not stated otherwise.

To assess the statistical properties of the observed and synthetic data, sets we use two robust statistical measures, the median and the median absolute deviation (MAD). The median is defined as a value in an ordered sample data set with an equal number of values above and below it. The MAD is defined as the median of the absolute deviations from the data set's median. We often give the MAD as a percentage of the median.

\subsection{Integrated intensities}

The most obvious criterion for statistical analyses of the spectral line profiles is the integrated intensity of the spectral lines. By comparing integrated intensities of the observed and synthetic spectra, we can easily distinguish the models with synthetic spectra considerably different from the observed one.

Figure 4 shows the comparison of the integrated intensity histograms of the observed (solid black lines) and synthetic Lyman- $\alpha$ to Lyman- $\delta$ line profiles. The histograms for three different models are displayed. Green dashed lines represent model VAR2, blue dash-dotted lines represent MoDEL1, and red dotted lines represent model VAR1. The $x$-axis gives the integrated intensities in erg $\mathrm{cm}^{-2} \mathrm{~s}^{-1} \mathrm{sr}^{-1}$. The $y$-axis represents the number of counts normalized to 1000 points in the data set (Counts $* 1000 / N$, where $N$ represents the total number of points in the data set). The inscription in each plot gives the median and the MAD as a percentage of the median for observed spectra and for models VAR2, MODEL1, and VAR1.

Histograms of models VAR2 and MODEL1 agree rather well with the histogram of the observed integrated intensities. In contrast, the histogram of the model VAR1, which differs from model VAR2 and MODEL1 only in the shape of the PCTR described by parameters $\gamma_{1}$ and $\gamma_{2}$, is considerably different from two other models and also from the observed spectra. We have also tested some additional models with different sets of the thread input parameters, and we have obtained even more pronounced deviations from the observed spectra than for model VAR1. 

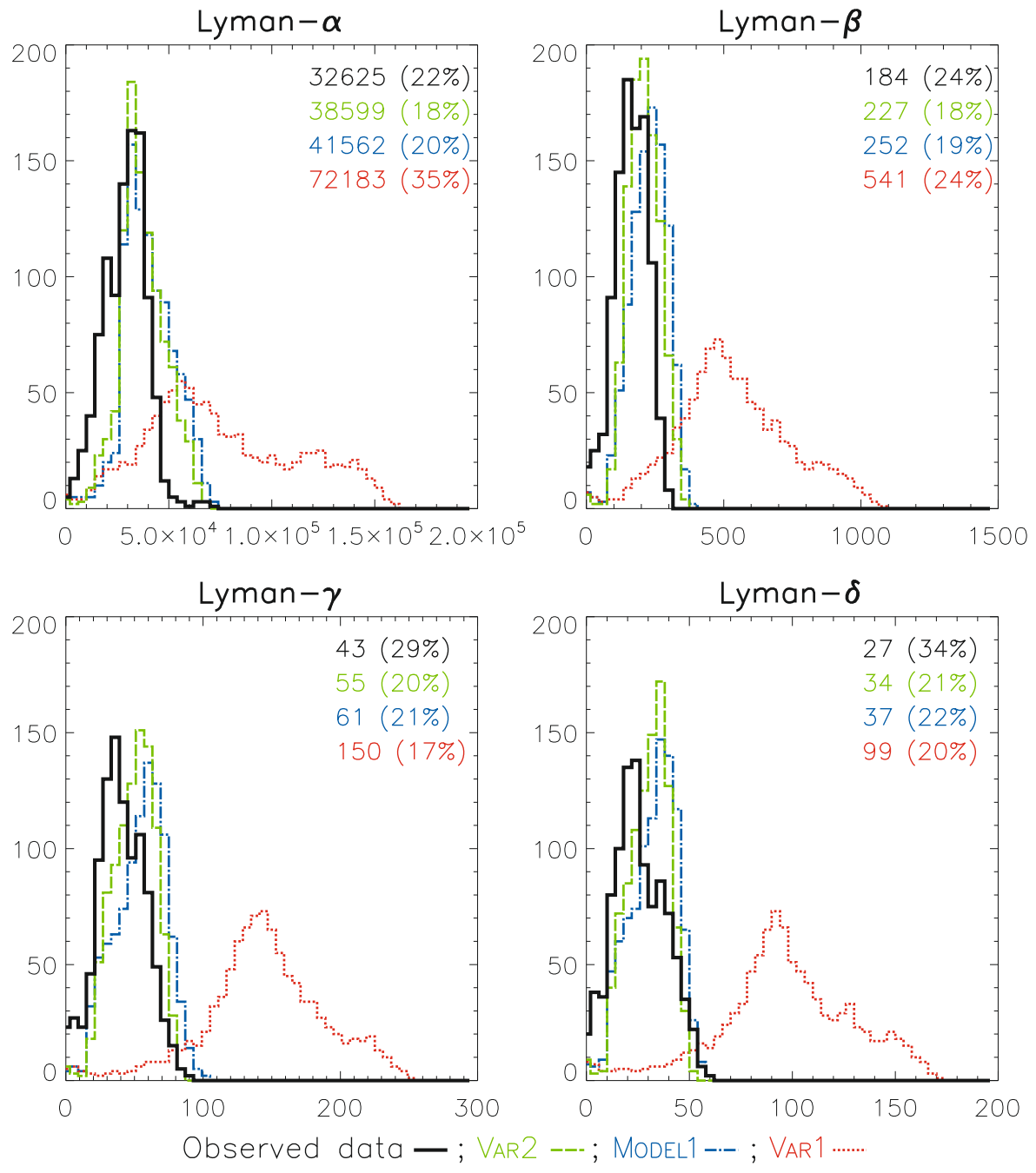

Fig. 4. Integrated intensity histograms of the observed and synthetic (model VAR2, MODEL1, and model VAR1) Lyman- $\alpha$ to Lyman- $\delta$ lines. The $x$-axis gives the integrated intensities in erg cm $\mathrm{cm}^{-2} \mathrm{~s}^{-1} \mathrm{sr}^{-1}$. The $y$-axis represents the number of counts normalized to 1000 points in the data set. The inscription in each plot gives the median and the MAD as a percentage of the median for the observed spectra and for models VAR2, MODEL1, and VAR1, respectively.

We have also analysed the effect of the choice of the LOS velocity intervals on the statistics of the integrated intensities. For this purpose we used the MODEL1 with three different intervals of the LOS velocities, $\langle-5,5\rangle,\langle-10,10\rangle$, and $\langle 20,20\rangle \mathrm{km} \mathrm{s}^{-1}$. The effect on the integrated intensity statistics of the synthetic Lyman line profiles is negligible.

Furthermore, we studied the effect of the LOS inclination, which also proved to be relatively small for the inclinations of up to $60^{\circ}$ from the LOS perpendicular to the magnetic field.

\subsection{Lyman decrement}

The Lyman- $\alpha$ to Lyman- $\beta$ integrated intensity ratio is quite sensitive to the geometrical and thermodynamical properties of the prominence fine structures. Even more so is the whole series of the Lyman- $\alpha$, Lyman- $\gamma$, and Lyman- $\delta$ to Lyman- $\beta$ ratios, known as the Lyman decrement. Therefore the Lyman decrement represents a very useful criterion for statistical comparison of the observed and synthetic spectra.

Figure 5 shows the Lyman decrement histograms of the integrated intensity ratios of the Lyman- $\alpha$, Lyman- $\gamma$, and Lyman- $\delta$ to the Lyman- $\beta$. Histograms of the observed and three sets of the synthetic spectra are shown. The $x$-axis gives the ratio of integrated intensities of the respective Lyman lines.

The histograms and their respective median values representing models VAR2 and MODEL1 are in very good agreement with the observed spectra. Especially in case of Lyman- $\alpha$ to Lyman- $\beta$, and to some extent also Lyman- $\gamma$ to Lyman- $\beta$ ratio, even the shape of the histograms represents the observed spectra rather well. In contrast, model VAR1 gives results that significantly differ from the observed data set throughout the whole Lyman decrement in both the shape of the histograms and their corresponding medians. Additional test models show even more pronounced deviations from the observed spectra than model VAR1.

The choice of the LOS velocity intervals has only a negligible effect on the statistics of the Lyman decrement. However, the inclination of the LOS with respect to that perpendicular to the magnetic field has a tangible effect on the Lyman decrement statistics, especially on the Lyman- $\alpha$ to Lyman- $\beta$ ratio. This seems in contrast with the statistics of the integrated intensities where the effect of the LOS inclination is negligible for all Lyman lines (Sect. 4.1). However, one needs to consider that we compared the histograms of the integrated intensities in the first case, while in the second case we computed particular Lyman line ratios in each point of the spectra locally, and only then we did compare the histograms of the particular ratios. To study the effect of the LOS inclination on the Lyman decrement statistics we used the MoDEL1 with $0^{\circ}$ (blue dash-dotted lines in Fig. 5), $30^{\circ}$, and $60^{\circ}$ angle between the inclined LOS and that perpendicular to the magnetic field. Medians of the Lyman line integrated intensity ratios are given in Table 3 . In this case the median values of the synthetic spectra with the best agreement with the observed spectra are those with the 


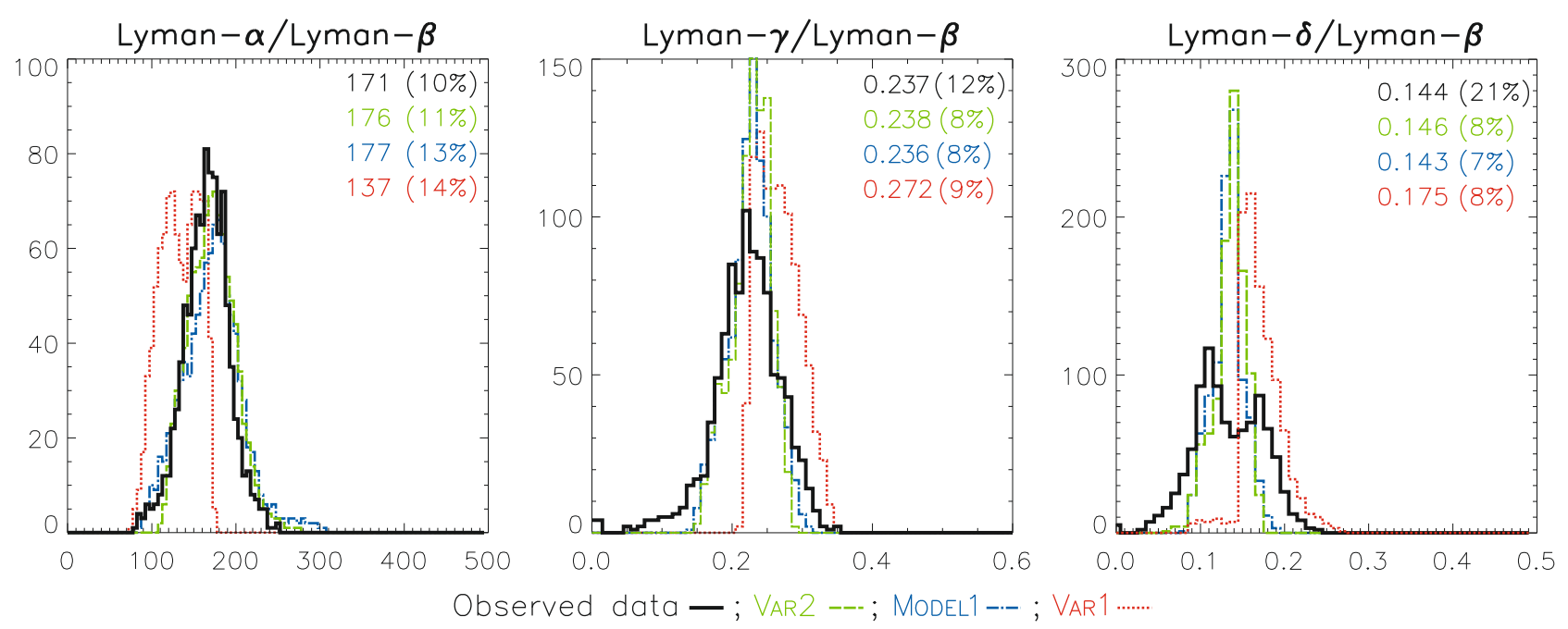

Fig. 5. Lyman decrement histograms of the observed spectra and synthetic spectra of models VAR2, MoDEL1, and VAR1. Panels show Lyman- $\alpha$ to Lyman- $\beta$, Lyman- $\gamma$ to Lyman- $\beta$, and Lyman- $\delta$ to Lyman- $\beta$ ratios, respectively. The $x$-axis gives the ratio of integrated intensities of the given Lyman lines. The $y$-axis represents the number of counts normalized to 1000 points in the data set. The inscription in each plot gives the median and the MAD as a percentage of the median for the observed spectra and for models VAR2, MODEL1, and VAR1.

Table 3. Median values of the Lyman line integrated intensity ratios for the MODEL1 with different LOS inclinations.

\begin{tabular}{cccc}
\hline \hline LOS inclination & $\frac{\text { Lyman- } \alpha}{\text { Lyman- } \beta}$ & $\frac{\text { Lyman- } \gamma}{\text { Lyman }-\beta}$ & $\frac{\text { Lyman- } \delta}{\text { Lyman }-\beta}$ \\
\hline $0^{\circ}$ & 177 & 0.236 & 0.143 \\
$30^{\circ}$ & 166 & 0.241 & 0.141 \\
$60^{\circ}$ & 153 & 0.252 & 0.139 \\
\hline
\end{tabular}

LOS perpendicular to the magnetic field. This might suggest the actual orientation of the LOS during the observations, but more profound investigation of the effects of the LOS inclination is needed.

\subsection{Red to blue peak intensity ratio}

The significant asymmetries of the observed Lyman line profiles in prominences are a well-known fact (e.g. Heinzel et al. 2001; Vial et al. 2007). Gunár et al. (2008) uses the multi-thread method for modelling the prominence fine structures to obtain the synthetic Lyman line profiles with asymmetries comparable to the observed ones. These authors show that even relatively low LOS velocities (of the order of $10 \mathrm{~km} \mathrm{~s}^{-1}$ ) can produce significantly asymmetrical Lyman line profiles. Such strong asymmetries cannot be attributed entirely to the effect of the Doppler shifts of the line profiles. Instead, as shown by Gunár et al. (2008), the asymmetrical profiles are produced by the combined effect of various Doppler shifts of the emission line profiles and the absorption coefficient profiles (optical thickness profiles) of the individual threads, together with relatively steep gradients of the absorption profiles and steep increase in intensity inside the line core. Gunár et al. (2008) also showed that such multi-thread fine structure models can produce Lyman- $\alpha$ line profiles with an asymmetry opposite to that of the higher Lyman lines at the same position. Such opposite asymmetries of different Lyman lines at the same position in the observed prominence were noticed by e.g. Vial et al. (2007).

Figures 6 and 7 show histograms of the ratios of the specific intensities in the peaks of the Lyman- $\alpha$ and Lyman- $\beta$, and
Lyman- $\gamma$ and Lyman- $\delta$ line profiles, respectively. The left panels of both figures display the specific intensity ratios of the red to dominant BLUE peaks, while the right panels show the ratios of the blue to dominant RED peaks. The $x$-axis gives the ratio of the specific intensities in the respective Lyman line profile peaks.

Figures 6 and 7 show that models VAR2, MODEL1, and VAR1 represent the overall pattern of the observed spectra histograms reasonably well; however, they fail to reproduce the strong predominance of the red peaks over the blue peaks of the Lyman- $\alpha$ and Lyman- $\beta$ line profiles. The histograms of the ratios of the specific intensities in the peaks are not affected by the shape of the PCTR of the individual threads of the multi-thread models.

On the other hand, the choice of the LOS velocity intervals has a significant effect on the statistics of the profile asymmetries. Figures 8 and 9 again show histograms of the ratios of the specific intensities in the peaks of the Lyman- $\alpha$ and Lyman$\beta$, and Lyman- $\gamma$ and Lyman- $\delta$ line profiles. The left hand panels display the specific intensity ratios of the red to dominant BLUE peaks and the right hand panels show the ratios of the blue to dominant RED peaks. Instead of VAR2 and VAR1 we show here two variations of the MODEL1 with LOS velocities from the interval $\langle-5,5\rangle$ marked as MoDEL1_v5 and from the interval $\langle-10,10\rangle$ marked as MODEL1. MODEL1_v5 gives better agreement with the observed spectra histogram for Lyman- $\alpha$ and Lyman- $\beta$ lines, but not as good agreement with higher Lyman lines. However, MODEL1_v20 differs from the observed spectra.

Table 4 gives the total number of profiles with the given asymmetry (dominant blue and red peak, respectively) for the observed and synthetic spectra. These numbers show the predominance of the red or blue asymmetry in the given data set. They can also indirectly indicate the occurrence of the opposite asymmetries of different Lyman lines at the same position in the prominence, when the predominance of one asymmetry for a given Lyman line changes to the predominance of the opposite asymmetry for another Lyman line. Such behaviour is in agreement with the findings of Vial et al. (2007) and Gunár et al. (2008) for observed and synthetic prominence Lyman spectra, respectively. 


\section{Lyman $-\alpha$}

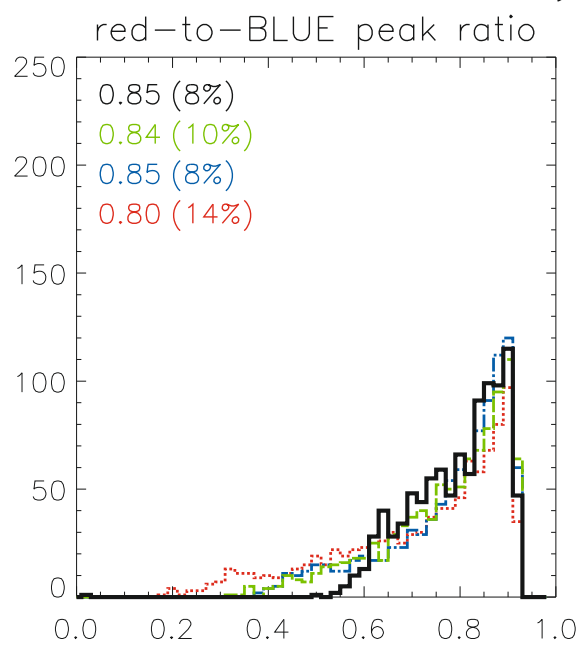

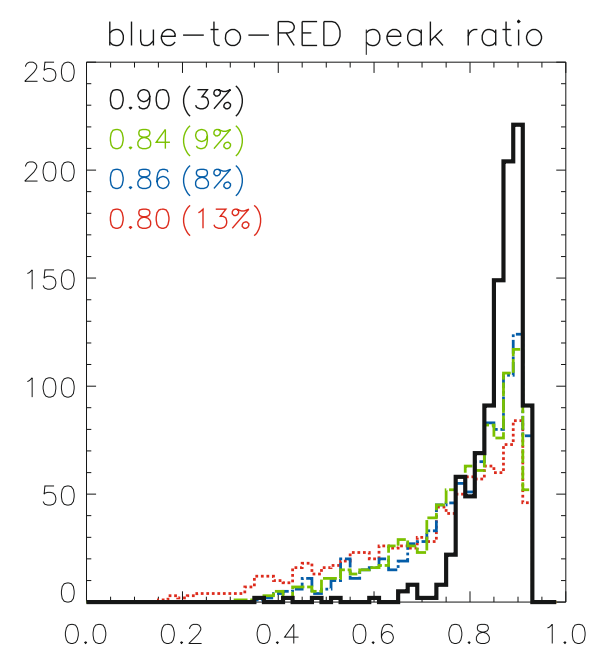

\section{Lyman $-\beta$}

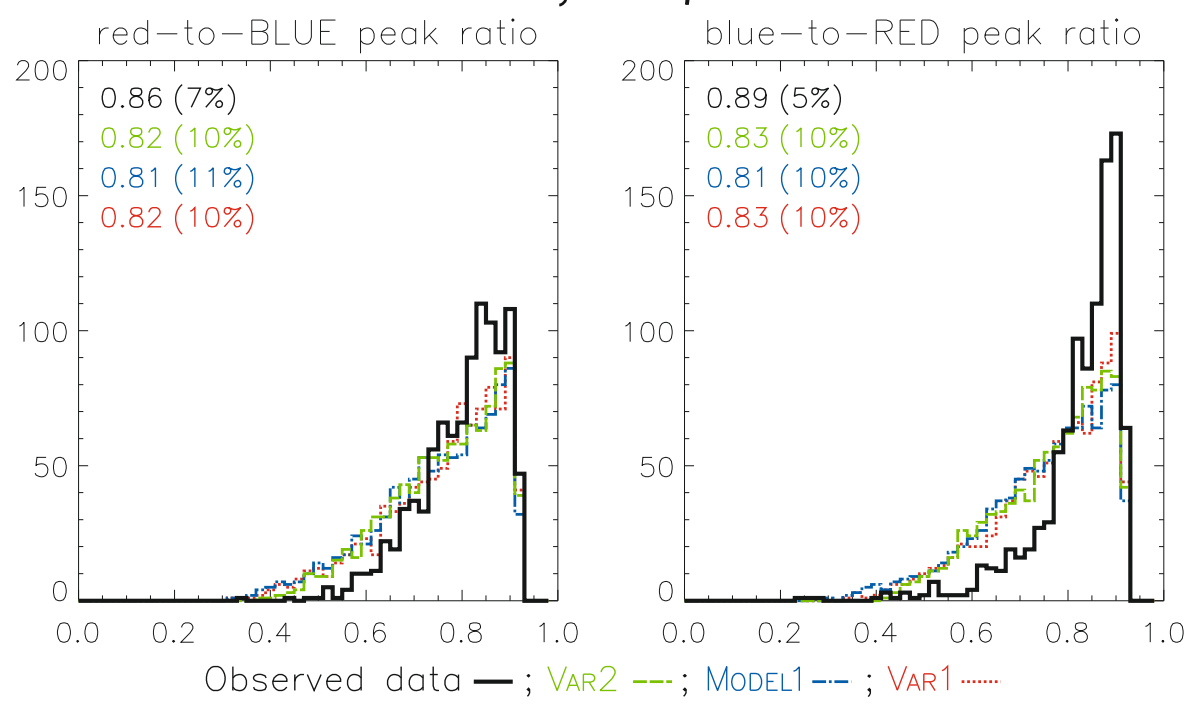

Fig. 6. Histograms of the ratios of the specific intensities of the red to dominant BLUE peak (left panels) and blue to dominant RED peak (right panels). The observed and the synthetic (model VAR2, MODEL1, and model VAR1) Lyman- $\alpha$ and Lyman- $\beta$ spectra are shown. The $x$-axis gives the ratio of the specific intensities in the given Lyman line peaks. The $y$-axis represents the number of counts normalized to 1000 points in the data set. The inscription in each plot gives the median, and the MAD as a percentage of the median for the observed spectra and for models VAR2, MODEL1, and VAR1.
Table 4. Total number of profiles with the given asymmetry for the observed and synthetic spectra.

\begin{tabular}{cccccccccc}
\hline \hline \multirow{2}{*}{ Models } & \multicolumn{2}{c}{ Lyman- $\alpha$} & \multicolumn{2}{c}{ Lyman- $\beta$} & \multicolumn{2}{c}{ Lyman- $\gamma$} & \multicolumn{2}{c}{ Lyman- $\delta$} \\
& blue & red & blue & red & blue & red & blue & red \\
\hline $\begin{array}{c}\text { Observed } \\
\text { data }\end{array}$ & 762 & 361 & 922 & 809 & 651 & 940 & 824 & 664 \\
MoDEL1 & 1906 & 2062 & 2403 & 2959 & 2164 & 2201 & 1886 & 2278 \\
VAR1 & 2669 & 2820 & 2631 & 2976 & 2359 & 2278 & 1891 & 2060 \\
VAR2 & 2327 & 2228 & 2631 & 2917 & 2354 & 2102 & 1989 & 2153 \\
$\begin{array}{c}\text { MODEL1 } \\
\text { _V5 }\end{array}$ & 1580 & 1429 & 2205 & 2377 & 2065 & 1486 & 1759 & 1647 \\
$\begin{array}{c}\text { MODEL1 } \\
\text { _V20 }\end{array}$ & 2111 & 2309 & 2679 & 3029 & 2485 & 2245 & 1922 & 2324 \\
\hline
\end{tabular}

The effect of the inclination of the LOS with respect to the normal to the magnetic field is relatively small; however, the increasing angle between the LOS and the normal to the magnetic field produces a slightly lower number of asymmetrical profiles. Thus the increase in the angle of the LOS and the normal to the magnetic field has a complementary effect to that of a lowering of the LOS velocity interval, as one would expect.

\subsection{Centre to mean-peak intensity ratio}

The last statistical criterion we employed in our statistical comparison of the observed and synthetic spectra is the depth of the reversal of the Lyman line profiles, characterized by the ratio of the specific intensity in the centre of the line to the averaged intensity of two peaks.

Figure 10 shows the histograms of the centre to the mean peak intensity ratios of the Lyman- $\alpha$ to Lyman- $\delta$ lines. The $x$-axis gives the ratio of the centre to mean peak specific intensity.

For the Lyman- $\gamma$ and Lyman- $\delta$ lines, the histograms of the observed and synthetic spectra are in relatively good agreement. However, the synthetic spectra are unable to reproduce the observed spectra histograms of the Lyman- $\alpha$ and Lyman- $\beta$ lines. Therefore, the synthetic Lyman- $\alpha$ and Lyman- $\beta$ line profile reversals are much deeper than the observed ones. Because the central intensities of the observed and synthetic line profiles agree rather well, the depth of the synthetic line profile reversals is due to very high peak intensities. 


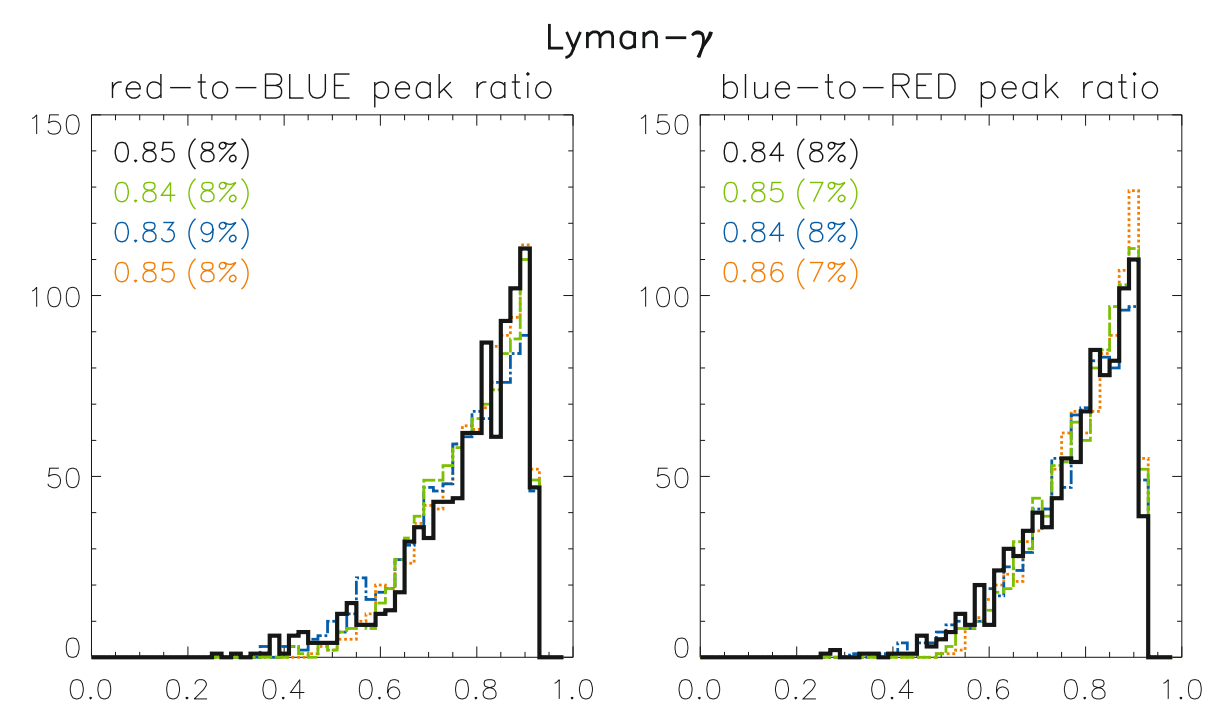

Lyman $-\gamma$

Lyman- $\delta$

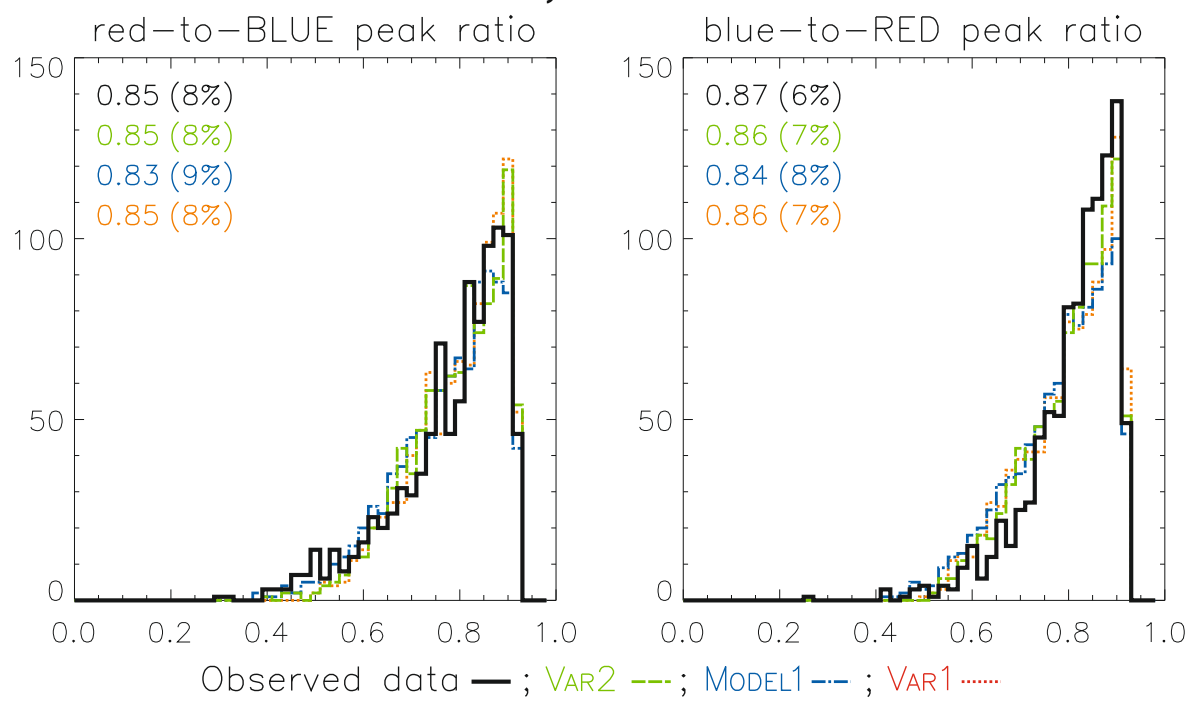

Fig. 7. Same as in Fig. 6, except that here we show the Lyman- $\gamma$ and Lyman- $\delta$ lines.

Unfortunately, we did not find any combination of the input parameters of the multi-thread models that would reproduce the observed spectra histograms. Nor has the LOS inclination any effect on the height of the peaks of the Lyman line profiles.

\section{Discussion}

We used four criteria well-suited to statistical analyses of the Lyman spectra of the prominences. Using these statistical criteria, we analysed a unique, extensive data set of observed Lyman lines, including the Lyman- $\alpha$ line obtained outside the SUMER attenuator. We compared the observed spectra with synthetic Lyman spectra obtained from 2D multi-thread models of the prominence fine structure with randomly assigned LOS velocities (Gunár et al. 2008).

Although it was not the purpose of this paper to find a multithread model with the best match of the synthetic and observed Lyman spectra, we have demonstrated very good agreement between the observed spectra and the synthetic spectra resulting from MoDEL1. The set of input parameters of the individual 2D thread of the multi-thread MoDEL1 (see Table 2) was obtained by Gunár et al. (2007), who used a trail-and-error method to find the model with the best agreement between the actual shape of the synthetic and observed Lyman line profiles. These authors used a part of the observed spectra also analysed in the present study. The approach of Gunár et al. (2007) of matching the shapes of individual synthetic Lyman line profiles with the observed profiles is significantly different from the analysis of the statistical criteria applied to all the data sets of synthetic and observed Lyman spectra.

The statistical criteria we have employed in this study are sensitive to the range of the input parameters of the $2 \mathrm{D}$ multithread models, so they represent a useful tool for assessing the properties of the prominence fine structures. The integrated intensities of the Lyman lines (Sect. 4.1) are suitable for analysis of the overall physical parameters of individual 2D fine-structure threads and are especially sensitive to the shape of the PCTR (see Fig. 4) which is, in our case, described by parameters $\gamma_{1}$ and $\gamma_{2}$.

The Lyman decrement criterion (Sect. 4.2) is very sensitive to all physical parameters of the individual threads and also to the inclination of the LOS with respect to the perpendicular to the magnetic field. Thus it is useful for more precise estimation of the physical parameters of the individual 2D threads 


\section{Lyman $-\alpha$}
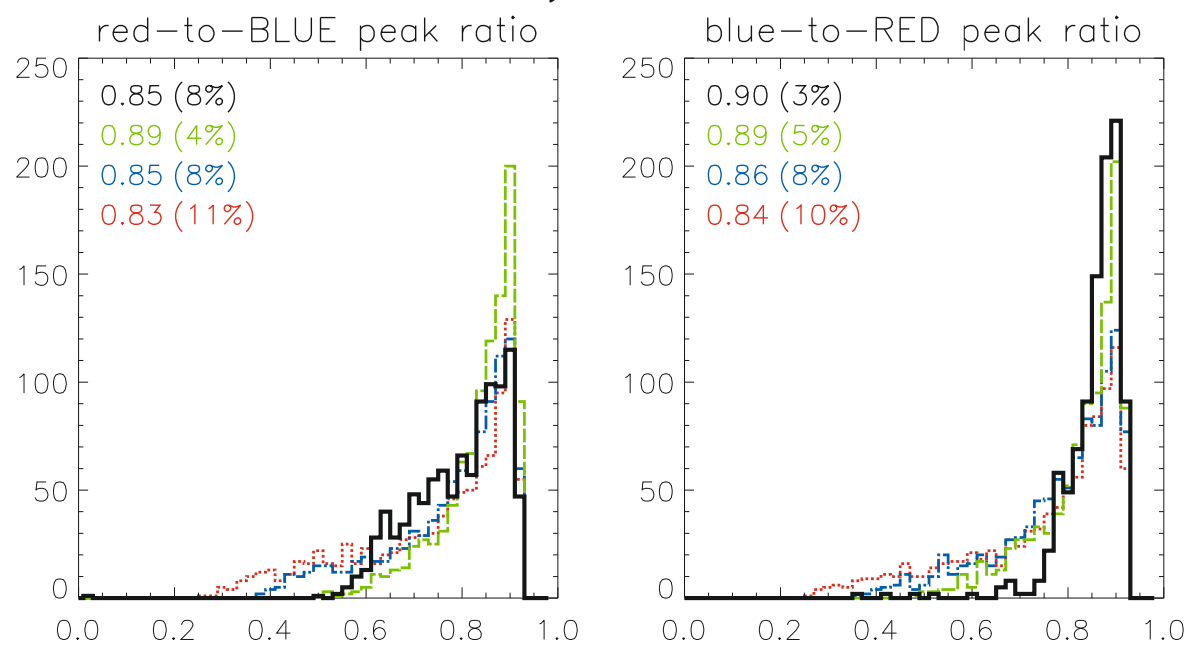

\section{Lyman $-\beta$}

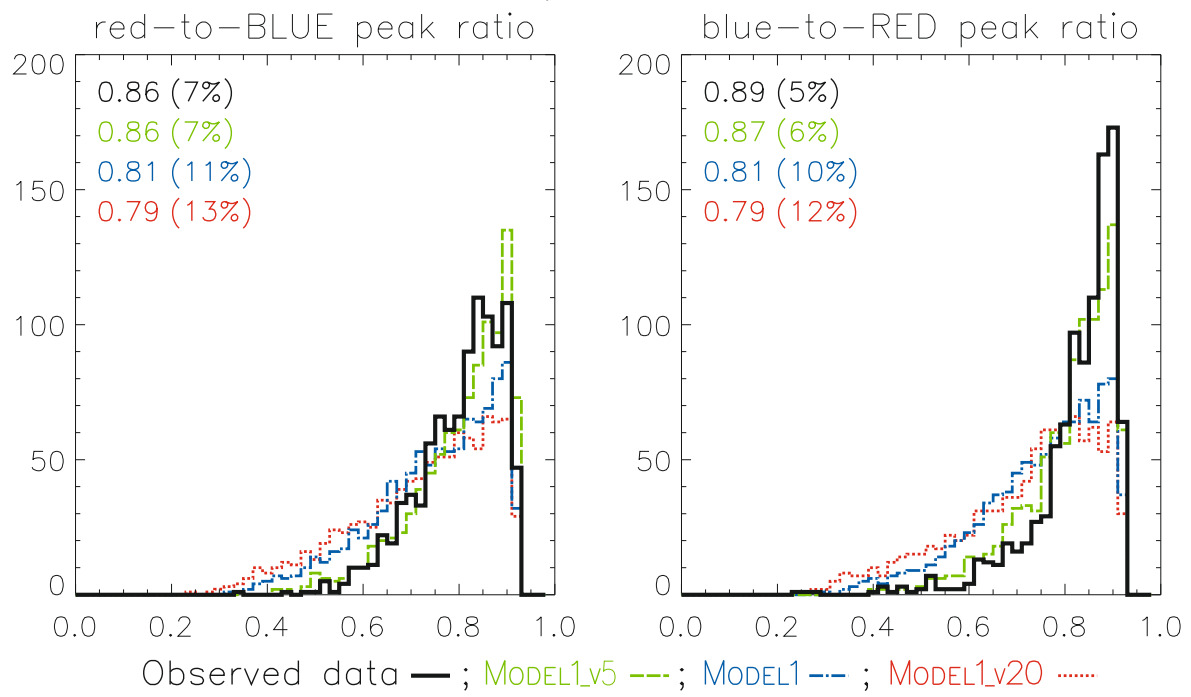

Fig. 8. Same as in Fig. 6, except that here green dashed lines represent MODEL1 with LOS velocities from the interval $\langle-5,5\rangle$ (marked MODEL1_v5), blue dash-dotted lines represent MODEL1 with LOS velocities from the interval $\langle-10,10\rangle$ (marked MODEL1), and red dotted lines represent MODEL1 with LOS velocities from the interval $\langle-20,20\rangle$ (marked MODEL1_v20). within multi-thread models and for determining the inclination of the LOS. The median values of the Lyman decrement ratios (see Fig. 5) of the MODEL1 are in very good agreement with observed spectra in all three studied ratios. This agreement has not been previously achieved (see e.g. Heinzel et al. 2001); however, no extensive statistical study has been done before. The Lyman- $\alpha$ to Lyman- $\beta$ ratio has recently been considered by Vial et al. (2007), who analysed a part of the same prominence studied in the present paper. The values of the observed Lyman- $\alpha$ to Lyman- $\beta$ ratio presented in Fig. 5 agree with values obtained by these authors and correspond to the earlier observations by the OSO-8 (see Vial 1982). The synthetic Lyman$\alpha$ and Lyman- $\beta$ integrated intensities were studied by Heinzel et al. (1987) using 1D non-LTE models of individual prominence slabs. These authors obtained low integrated intensities of the synthetic Lyman- $\beta$ line, which leads to large synthetic Lyman- $\alpha$ to Lyman- $\beta$ ratios in comparison with the observations. Similar results were obtained also by Gouttebroze et al. (1993) (see Table 5). Inclusion of the PCTR to the 1D prominence modelling (Anzer \& Heinzel 1999) led to higher Lyman- $\beta$ integrated intensities and thus to lower Lyman- $\alpha$ to Lyman- $\beta$ ratios. The Lyman- $\alpha$ to Lyman- $\beta$ ratio was also studied by Vial et al. (1989)
Table 5. Lyman- $\alpha$ to Lyman- $\beta$ integrated intensity ratios obtained by various prominence models.

\begin{tabular}{cc}
\hline \hline Type of models & Lyman- $\alpha /$ Lyman- $\beta$ \\
\hline 1D models without PCTR $^{1}$ & $90-400$ \\
1D models with PCTR $^{2}$ & $110-280$ \\
1D multi-slab models without A.D. $^{3}$ & $42-111$ \\
1D multi-slab models with A.D. $^{4}$ & $2.3-18.5$ \\
\hline
\end{tabular}

References. ${ }^{1}$ Gouttebroze et al. (1993); ${ }^{2}$ Anzer \& Heinzel (1999); ${ }^{3}$ Vial et al. (1989); ${ }^{4}$ Fontenla et al. (1996).

Notes. A.D. means ambipolar diffusion.

using 1D multi-slab models. Similar 1D multi-slab models were used by Fontenla et al. (1996), who also used the ambipolar diffusion. However, the resulting Lyman- $\alpha$ to Lyman- $\beta$ ratio starkly contrasts with the observed values (see also conclusions of Vial et al. 2007).

The asymmetries of the Lyman line profiles are represented by the red to blue peak intensity ratio criterion (Sect. 4.3). This is mostly sensitive to the choice of the LOS velocity intervals 


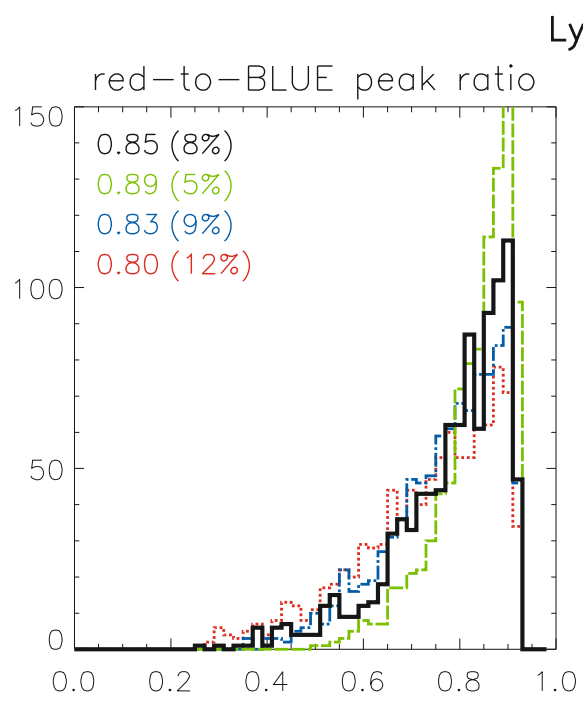

\section{Lyman $-\gamma$}

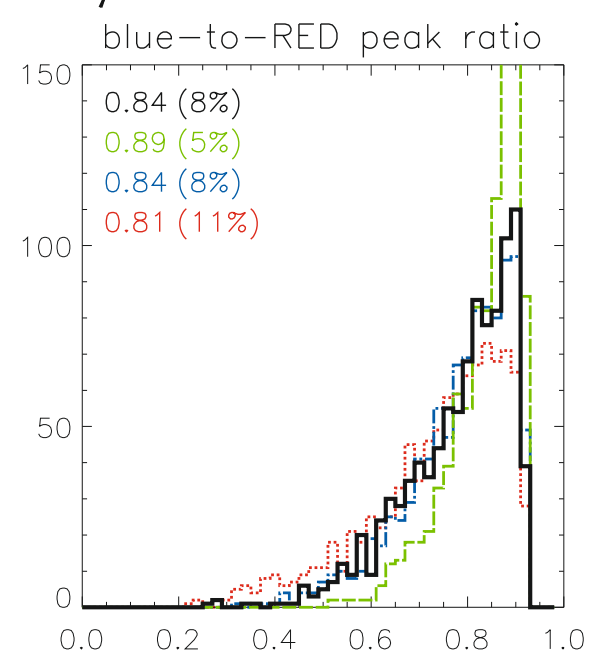

Lyman $-\delta$

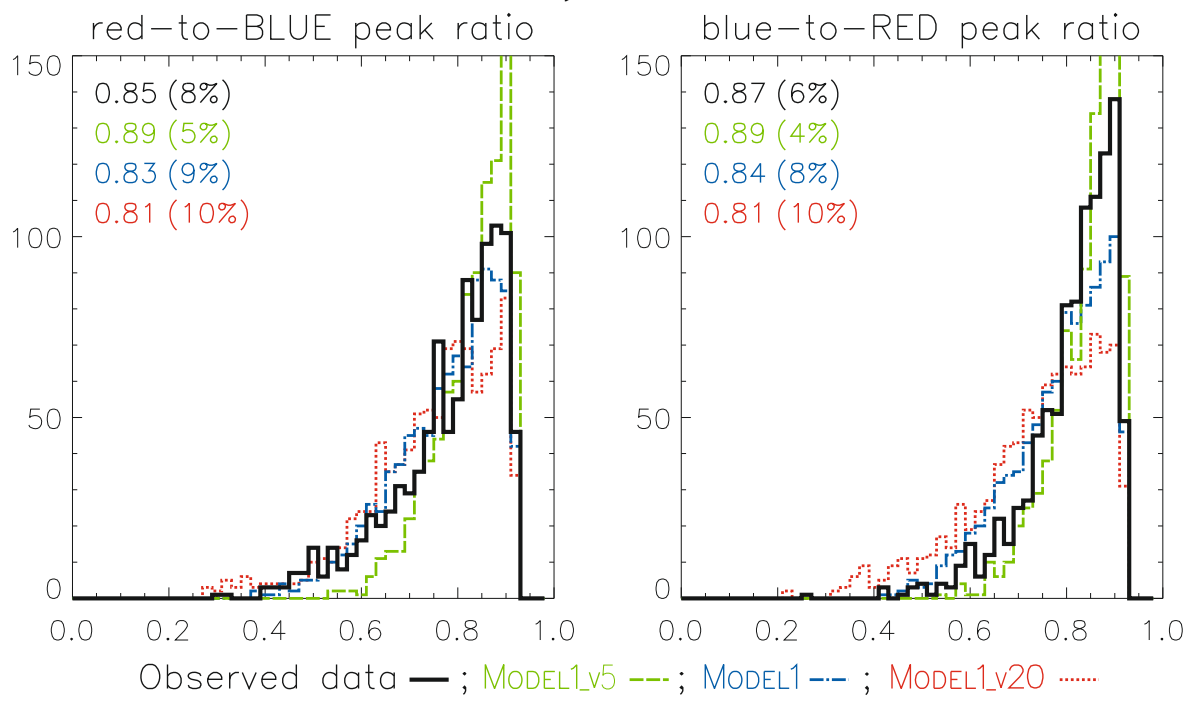

Fig. 9. Same as in Fig. 6, except that here we show the Lyman- $\gamma$ and Lyman- $\delta$ lines. Green dashed lines represent MODEL1 with LOS velocities from the interval $\langle-5,5\rangle$ (marked MoDEL1_v5), blue dash-dotted lines represent MODEL1 with LOS velocities from the interval $\langle-10,10\rangle$ (marked MoDEL1), and red dotted lines represent MoDEL1 with LOS velocities from the interval $\langle-20,20\rangle$ (marked MODEL1_v20).

(Figs. 8 and 9), so is useful for assessing of the LOS velocities of individual threads of the multi-thread models.

The depth of the reversed Lyman line profiles is described by the centre-to-mean-peak intensity ratio criterion (Sect. 4.4). The synthetic spectra obtained by multi-thread modelling are unable to reproduce the statistics of the observed Lyman spectra (Fig. 10). We did not succeed in finding a good fit to the observed spectra by any realistic combination of the input parameters of the multi-thread models. This comes from very high peak intensities compared to the observed profiles. The central intensities of the observed and synthetic profiles are in good agreement. Although the peak intensities of the synthetic line profiles are much higher than observed ones, the integrated intensities of the observed and synthetic lines are in good agreement, because of the sharpness of the synthetic line peaks and smaller line-width of the synthetic Lyman lines, compared to the observed spectra.

\section{Conclusions}

Our 2D multi-thread models of the prominence fine structures are able to produce synthetic Lyman spectra in very good agreement with the observed spectra when evaluated by a number of statistical criteria. They are also able to reproduce the shape of the observed Lyman line profiles in particular parts of the spectra. In masse, however, our synthetic Lyman line profiles exhibit rather sharp peaks with high intensities, which are not commonly found in the observed spectra. This is, in fact, a rather common phenomenon in modelling not only of prominences but also of the quite chromosphere and other solar atmospheric features. This problem could be caused by effects of the redistribution in the radiative transfer computations, by some effects of the $2 \mathrm{D}$ radiative transfer that might be addressed by full 3D modelling or by the effect of the mutual radiative interaction of individual threads of the multi-thread model.

Our preliminary tests of the effect of mutual radiative interaction on the synthetic Lyman line profiles show a slight enhancement of the peak intensities in comparison with models without mutual radiative interaction. This even more emphasizes the discrepancy between the intensity and sharpness of line peaks of the observed and the synthetic spectra. However, these tests were only performed on ensembles of 3 and 5 fine-structure threads without any LOS velocities. More realistic computations of the whole multi-thread models with LOS velocities and mutual radiative interaction are needed. However, such models are extremely time-consuming, because the whole multi-thread model needs to be calculated within one large computational box, which is not the case for our method. 

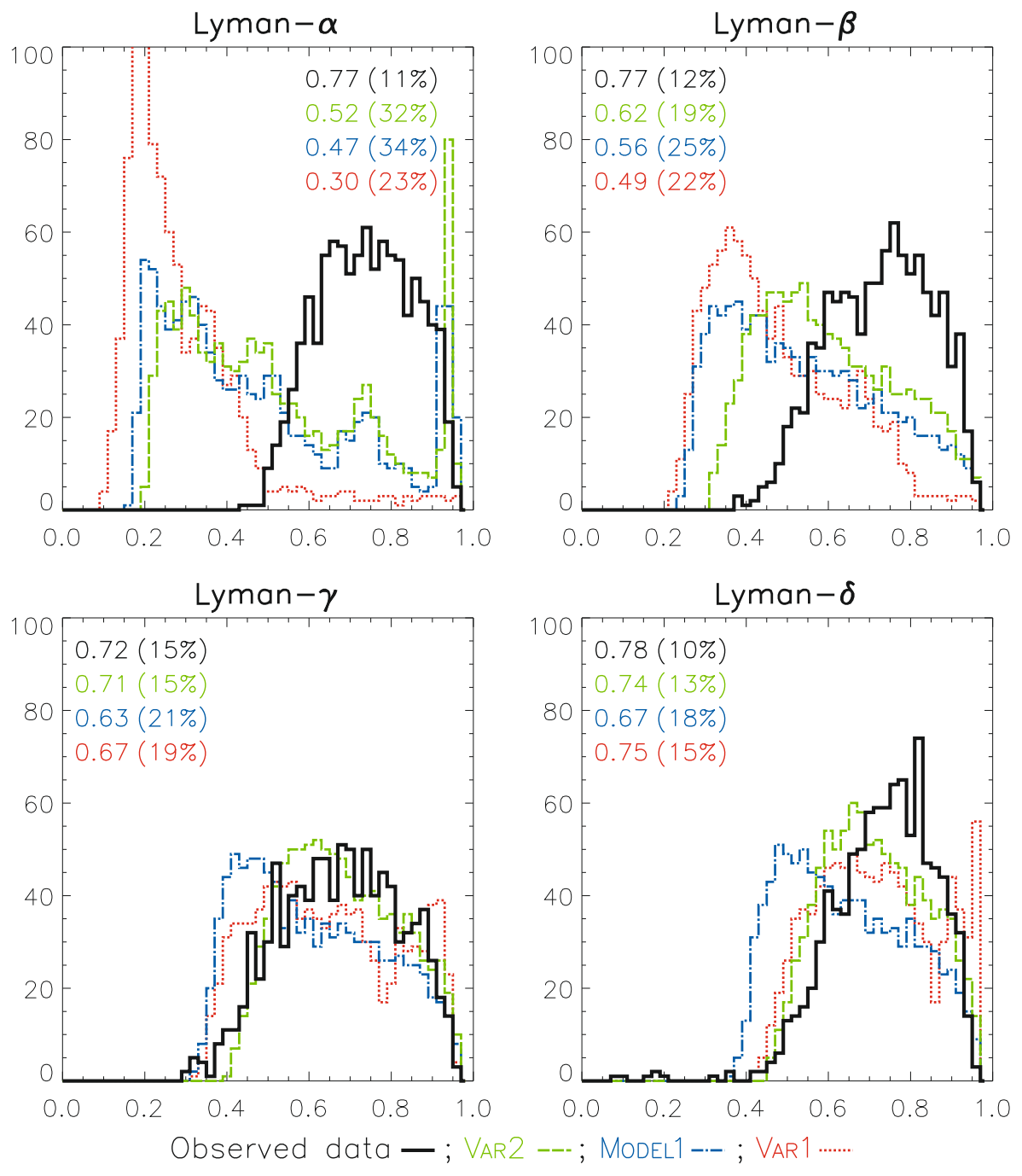

Fig. 10. Centre to mean peak intensity ratio histograms of the observed and synthetic (model VAR2, Model1, and model VAR1) Lyman- $\alpha$ to Lyman- $\delta$ line profiles. The $x$-axis gives the ratio of the centre to mean peak specific intensity. The $y$-axis represents the number of counts normalized to 1000 points in the data set. The inscription in each plot gives the median and the MAD as a percentage of the median for the observed spectra and for the models VAR2, MODEL1, and VAR1.

The solution of this phenomenon would represent an important step in modelling various solar atmospheric features. In achieving this, our set of statistical criteria could provide a useful test case because of its sensitivity to a number of different physical parameters.

The same method of employing a set of statistical criteria similar to our present study could also be used for analysing helium lines, as well as the spectral lines of other elements. In such a case one would need to use comprehensive atomic models of helium or any other species to obtain realistic synthetic spectra.

Acknowledgements. S.G. acknowledges support from grant 205/09/P554 of the Grant Agency of Czech Republic. S.G. and P.S. acknowledges support from grant 205/07/1100 of the Grant Agency of the Czech Republic. P.H. acknowledges the support from grant 1QS300120506 of the Grant Agency of the Academy of Sciences of the Czech Republic. This work was also supported by ESA-PECS project No. 98030 and the institutional project AV0Z10030501.

\section{References}

Anzer, U., \& Heinzel, P. 1999, A\&A, 349, 974

Chae, J., Schühle, U., \& Lemaire, P. 1998, ApJ, 505, 957
Curdt, W., Brekke, P., Feldman, U., et al. 2001, A\&A, 375, 591

Fontenla, J. M., Rovira, M., Vial, J.-C., \& Gouttebroze, P. 1996, ApJ, 466, 496 Gouttebroze, P., Heinzel, P., \& Vial, J. C. 1993, A\&AS, 99, 513

Gunár, S., Heinzel, P., Schmieder, B., Schwartz, P., \& Anzer, U. 2007, A\&A, 472, 929

Gunár, S., Heinzel, P., Anzer, U., \& Schmieder, B. 2008, A\&A, 490, 307

Harrison, R. A., Sawyer, E. C., Carter, M. K., et al. 1995, Sol. Phys., 162, 233

Heasley, J. N., \& Mihalas, D. 1976, ApJ, 205, 273

Heinzel, P. 2007, in The Physics of Chromospheric Plasmas, ed. P. Heinzel, I.

Dorotovič, \& R. J. Rutten, ASP Conf. Ser., 368, 271

Heinzel, P., \& Anzer, U. 2001, A\&A, 375, 1082

Heinzel, P., Anzer, U., \& Gunár, S. 2005, A\&A, 442, 331

Heinzel, P., Gouttebroze, P., \& Vial, J.-C. 1987, A\&A, 183, 351

Heinzel, P., Schmieder, B., Vial, J.-C., \& Kotrč, P. 2001, A\&A, 370, 281

Kippenhahn, R., \& Schlüter, A. 1957, ZAp, 43, 36

Mihalas, D., Auer, L. H., \& Mihalas, B. R. 1978, ApJ, 220, 1001

Patsourakos, S., \& Vial, J.-C. 2002, Sol. Phys., 208, 253

Schmieder, B., Gunár, S., Heinzel, P., \& Anzer, U. 2007, Sol. Phys., 241, 53

Schühle, U. 2003, SUMER Data Cookbook, Published on internet at http:// WWw.mps.mpg.de/projects/soho/sumer/text/cookbook.html

Vial, J. C. 1982, ApJ, 253, 330

Vial, J.-C., Ebadi, H., \& Ajabshirizadeh, A. 2007, Sol. Phys., 246, 327

Vial, J. C., Rovira, M., Fontela, J., \& Gouttebroze, P. 1989, Hvar Observatory Bulletin, 13, 347

Wilhelm, K., Curdt, W., Marsch, E., et al. 1995, Sol. Phys., 162, 189 